\title{
Clinical Validity of Next-Generation Sequencing Multi- Gene Panel Testing for Detecting Pathogenic Variants in Patients With Hereditary Breast-Ovarian Cancer Syndrome
}

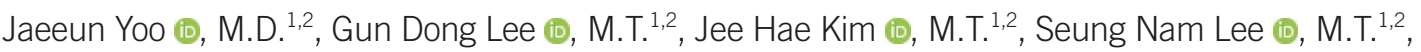

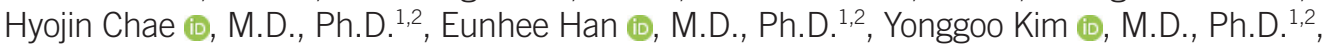 \\ and Myungshin Kim (i), M.D., Ph.D., \\ ${ }^{1}$ Department of Laboratory Medicine and ${ }^{2}$ Catholic Genetic Laboratory Center, Seoul St. Mary's Hospital, College of Medicine, The Catholic University of \\ Korea, Seoul, Korea
}

Background: Hereditary breast and ovarian cancer syndrome (HBOC) is caused by pathogenic variants in BRCA and other cancer-related genes. We analyzed variants in $B R C A$ gene and other cancer-related genes in HBOC patients to evaluate the clinical validity of next-generation sequencing (NGS) multi-gene panel testing.

Methods: The BRCA1/2 NGS testing was conducted for $262 \mathrm{HBOC}$ patients. Multiplex ligation-dependent probe amplification and direct Sanger sequencing were performed for confirmation. Multi-gene panel testing was conducted for 120 patients who did not possess BRCA1/2 pathogenic variants but met the National Comprehensive Cancer Network criteria.

Results: Pathogenic variants in BRCA1/2 were detected in $30 \mathrm{HBOC}$ patients (11.5\%). Additionally, four out of the 120 patients possessed pathogenic variants by multi-gene panel testing (3.3\%): MSH2 (c.256G > T, p.Glu86*), PMS2 (c.1687C>T, p.Arg563*), CHEK2 (c.546C >A, p.Tyr182*), and PALB2 (c.3351-1G>C). All the four patients had a family history of cancer.

Conclusions: Multi-gene panel testing could be a significant screening tool for HBOC patients, especially for those with a family history of cancer.

Key Words: Hereditary breast and ovarian cancer syndrome, BRCA1/2, Pathogenic variants, Multi-gene panel, Clinical validity, Next-generation sequencing
Received: December 5, 2018

Revision received: June 3, 2019

Accepted: October 11, 2019

\section{Corresponding author:}

Myungshin Kim, M.D., Ph.D.

Department of Laboratory Medicine,

College of Medicine, The Catholic

University of Korea, Seoul St. Mary's

Hospital, 222 Banpo-daero, Seocho-gu,

Seoul 06591, Korea

Tel: +82-2-2258-1645

Fax: +82-2-2258-1719

E-mail: microkim@catholic.ac.kr

\section{(c) (i) (8)}

(c) Korean Society for Laboratory Medicine This is an Open Access article distributed under the terms of the Creative Commons Attribution Non-Commercial License (http://creativecommons.org/licenses/by-nc/4.0) which permits unrestricted non-commercial use, distribution, and reproduction in any medium, provided the original work is properly cited.

\section{INTRODUCTION}

Hereditary breast and ovarian cancer syndrome ( $\mathrm{HBOC}$ ) is characterized by increased susceptibility to the development of breast, ovarian, and other cancers [1]. The significance of the BRCA1 and $B R C A 2(B R C A 1 / 2)$ germline variants as predictors of $\mathrm{HBOC}$ has been well established [2]. Identification of germline variants in BRCA1/2 has improved the prevention and diagnosis of $\mathrm{HBOC}$ [3]. However, BRCA1/2 pathogenic variants have been observed in only approximately $20 \%$ of suspected hereditary cases [4]; several other cases are suspected to be caused by germline pathogenic variants in other cancer-related genes [5].

Recent advances in genetic testing have led to the discovery of many genes that increase the susceptibility to cancer [5]. Fur- 
thermore, the development of next-generation sequencing (NGS) has enabled simultaneous testing of multiple genes. Many recent studies have examined the clinical validity of comprehensive multi-gene panel testing in breast and ovarian cancers [68]. It has been reported that $3 \%$ to $4 \%$ of high-risk patients have pathogenic variants in cancer-related genes other than BRCA1/2, but the exact prevalence details remain unknown $[9,10]$. Therefore, clear clinical management guidelines should be established, and evidence-based clinical validity of multi-gene panel testing should be conducted. In this study, we evaluated the clinical validity of multi-gene panel, including BRCA1/2 and 25 other cancer-related genes as a screening tool for patients with HBOC.

\section{METHODS}

\section{Study population}

This was a retrospective study based on chart review and test results. We reviewed 262 patients with breast or ovarian cancer who underwent BRCA1/2 gene testing from May 2015 to July 2017 in Seoul St. Mary's Hospital, Seoul, Korea and met the National Comprehensive Cancer Network (NCCN) criteria for HBOC [11]. For inclusion, $\mathrm{HBOC}$ should be suspected in individuals with a personal or family history of any of the followings: (a) breast cancer diagnosed at or before the age of 45 years; (b) ovarian cancer; (c) multiple primary breast cancers either in one or both breasts; (d) triple-negative (estrogen receptor-negative, progesterone receptor-negative, and human epidermal growth factor receptor 2 [HER2/neu]-negative) breast cancer, particularly when diagnosed before the age of 60 years; (e) two or more relatives with breast cancer, with at least one under the age of 50 years; (f) three or more relatives with breast cancer at any age; and (g) a previously identified $B R C A 1$ or $B R C A 2$ pathogenic variant in

Table 1. Clinical characteristics of breast and ovarian cancer patients $(N=262)$

\begin{tabular}{lccc}
\hline & $\begin{array}{c}\text { Breast cancer } \\
(\mathrm{N}=132)\end{array}$ & $\begin{array}{c}\text { Ovarian cancer } \\
(\mathrm{N}=125)\end{array}$ & $\begin{array}{c}\text { Breast and ovarian } \\
\text { cancer }(\mathrm{N}=5)\end{array}$ \\
\hline $\begin{array}{l}\text { Age at diagnosis (yr), } \\
\text { median (range) }\end{array}$ & $41.5(26-75)$ & $51(19-89)$ & $47(41-55)$ \\
$\begin{array}{l}\text { Family history } \\
\text { Yes, } \mathrm{N}(\%)\end{array}$ & $32(24.2)$ & $39(31.2)$ & $1(20.0)$ \\
No, N (\%) & $100(75.8)$ & $86(68.8)$ & $4(80.0)$ \\
$\begin{array}{l}\text { BRCA1/2 pathogenic } \\
\text { variant-positive, } \mathrm{N}(\%) \\
\text { BRCA1 }\end{array}$ & $15(11.4)$ & $13(10.4)$ & $2(40)$ \\
BRCA2 & 5 & 10 & 2 \\
\hline
\end{tabular}

the family. Medical and family histories were provided by the referring physicians and/or were collected through a review of the patients' medical records (Table 1 ).

The BRCA1/2 test results of the 262 patients were evaluated. According to the $B R C A$ testing strategy of our institution, patients who tested negative in the BRCA1/2 test but met the NCCN multigene testing criteria underwent cancer-related multi-gene panel testing (Fig. 1). The NCCN multi-gene testing criteria were as follows: (a) at least one case of breast or ovarian cancer in a firstor second-degree relative; (b) bilateral breast cancer; (c) a first diagnosis of breast cancer before the age of 40 years; and (d) codiagnosis of breast and ovarian cancers in the same patient. In total, $120 \mathrm{HBOC}$ patients were selected for the NGS multi-gene panel testing targeting cancer-related genes other than BRCA1/2.

Multi-gene panel testing was performed for 120 of the 232 BRCA1/2-negative patients, including 72 breast cancer patients, 47 ovarian cancer patients, and one patient with both breast and ovarian cancers. All patients previously provided informed consent for all tests, including the BRCA1/2 gene and multigene panel testing. This study was approved by the Institutional Review Board/Ethics Committee of Seoul St. Mary's Hospital, Seoul, Korea (KC15RISI0915).

\section{Sample collection and preparation}

Peripheral blood samples were collected from HBOC patients. Genomic DNA was extracted from whole blood using the QIAamp

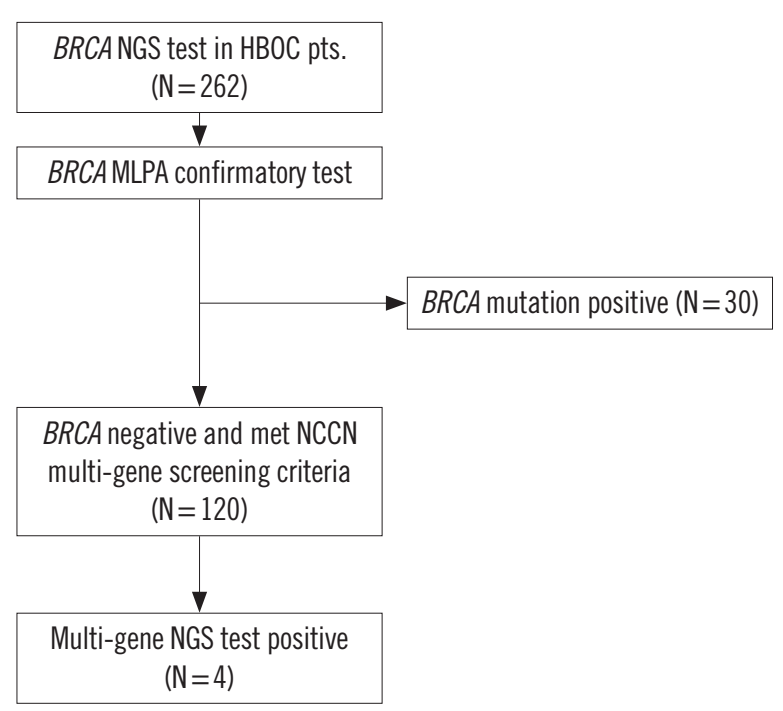

Fig. 1. Scheme of $B R C A 1 / 2$ and multi-gene NGS testing. Abbreviations: BRCA, BReast CAncer gene; NGS, next-generation sequencing; HBOC, hereditary breast and ovarian cancer; Pts, patients; MLPA, multiplex ligation-dependent probe amplification; NCCN, National Comprehensive Cancer Network. 
DNA Blood Mini Kit (Qiagen, Venlo, The Netherlands). Each DNA sample was checked for purity using a NanoDrop 1000 system (Thermo Fisher Scientific, Rockford, IL, USA), and DNA concentration was determined using a Qubit 3.0 fluorometer (Thermo Fisher Scientific). Extracted DNA was stored at $-80^{\circ} \mathrm{C}$ until further use.

\section{BRCA $1 / 2$ variant testing using NGS}

The library was prepared using the Ion Chef System (Thermo Fisher Scientific) according to the manufacturer's instructions. Briefly, barcoded libraries were generated from 10 ng of DNA per sample using the lon AmpliSeq Chef Solutions DL8 Kit (Thermo Fisher Scientific) and the Oncomine BRCA Research Assay (Thermo Fisher Scientific). Two premixed pools of 265 primer pairs were used to generate the sequencing libraries. Clonal amplification of the libraries was performed by emulsion PCR using an Ion AmpliSeq IC 200 Kit (Thermo Fisher Scientific). Next, the prepared libraries were sequenced on an lon S5 XL Sequencer using an lon 520 Chip and an lon 520 kit-Chef Kit (Thermo Fisher Scientific). Raw sequence data in FASTQ format were aligned to the hg19 human reference genome using the Torrent Mapping Alignment Program aligner implemented in v5.2 of the Torrent Suite software (Thermo Fisher Scientific). We used the plug-in Torrent Variant Caller v5.2.0.34 (Thermo Fisher Scientific) for single nucleotide variant (SNV) calling to generate a variant call format file. For Torrent Variant Caller analysis, the default settings of the germline low-stringency parameters (minimal variant frequency, 0.1 ; minimum variant quality, 10; minimum coverage, $5 \times$; maximum strand bias, 0.98; and minimum variant score, 10) were used and candidate variants were obtained only at a variant frequency at a given position $\geq 20 \%$ and variant coverage $\geq 20 \times$. Copy number variation (CNV) and insertions and deletions (indels) were evaluated using a single integrated workflow with the Ion PGM platform (Thermo Fisher Scientific, San Jose, CA, USA).

\section{Multiplex ligation-dependent probe amplification analysis (MLPA) for CNV}

Following BRCA1/2 NGS analysis, MLPA was conducted for BRCA1/2 (MRC-Holland, Amsterdam, The Netherlands) to identify or confirm any large genomic rearrangements (LGR). The inclusion criteria were: (a) early-onset breast cancer (diagnosed at $\leq 36$ years); (b) two breast primaries (include bilateral disease or two or more ipsilateral primary tumors); (c) breast cancer diagnosed at any age, with $\geq$ one close blood relatives (including first-, second-, or third-degree) with breast and/or epi- thelial ovarian cancer; (d) both breast and epithelial ovarian cancers diagnosed at any age; and (e) epithelial ovarian cancer with $\geq$ one close blood relatives with breast and/or epithelial ovarian cancer, according to our previous study [12]. The MLPA results were analyzed using GeneMarker software (Softgenetics, State College, PA, USA). Peak heights were normalized, and deletions or duplications were defined as recommended by the manufacturer. Direct sequencing of the probe binding and ligation sites was performed for the relevant exons to detect nearby variants, which can lead to a false decrease in peak signal.

\section{Multi-gene panel testing using NGS}

Following BRCA1/2 variant testing, we conducted a multi-gene panel testing using NGS with a targeted capture sequencing panel (BRCA Reflex, Hereditary Cancer Research Panel, Thermo Fisher Scientific Inc., Waltham, MA, USA) that included all coding sequences and intron-exon boundaries of the coding exon from 25 hereditary cancer-related genes with a central role in DNA repair or the mismatch repair (MMR) pathway (APC, ATM, BARD1, BMPR1A, BRIP1, CDH1, CDK4, CDKN2A, CHEK2, EPCAM, MLH1, MRE11A, MSH2, MSH6, MUTYH, NBN, PALB2, PMS2, PTEN, RAD50, RAD51C, RAD51D, SMAD4, STK11, and TP53) [13]. NGS analysis was subsequently performed using the Ion Torrent S5 system (Life Technologies, Foster City, CA, USA). Highquality sequencing data with an average depth of 500-1,000-folds were acquired. Annotation of SNVs, indels, and splice-site alterations was performed using the lon Reporter Server System (Life Technologies).

\section{Confirmatory sanger sequencing}

The detected pathogenic and likely pathogenic variants identified by NGS analysis were verified by Sanger sequencing. Direct sequencing of entire coding exons and flanking intronic sequences of relevant genes was performed bi-directionally on an $A B I$ 3130XL Genetic Analyzer (Applied Biosystems, Foster City, CA, USA) with a BigDye Terminator v3.1 Cycle Sequencing Kit (Applied Biosystems). Chromatograms were analyzed with Sequencher software version 5.0 (Gene Codes, Ann Arbor, MI, USA). Sanger sequencing was performed as described previously [14]. Exon numbering and DNA sequence variant descriptions are based on NM_007294.3 and NM_000059.3 as reference sequences for BRCA1/2. The reference sequences for CHEK2, MLH1, MSH2, PALB2, and PMS2 are as follows: CHEK2 NM_ 001005735.1, MLH1 NM_000249.3, MSH2 NM_000251.2, PALB2 NM_024675.3, and PMS2 NM_000535.6. 


\section{Genetic variant classification}

Genetic variants were classified using a five-tier system according to the guidelines of the American College of Medical Genetics and Genomics (ACMG): pathogenic, likely pathogenic, variant of unknown significance (VUS), likely benign, or benign [15]. Pathogenic and likely pathogenic variants were considered significant.

\section{Statistical analysis}

Normally distributed continuous variables were summarized as mean \pm SD and compared using the Student t-test. Patient age was summarized as median (range). All tests were two-tailed. $P \leq 0.05$ was considered statistically significant. Statistical analyses were performed using MedCalc version 15.5 (MedCalc, Ostend, Belgium).
A
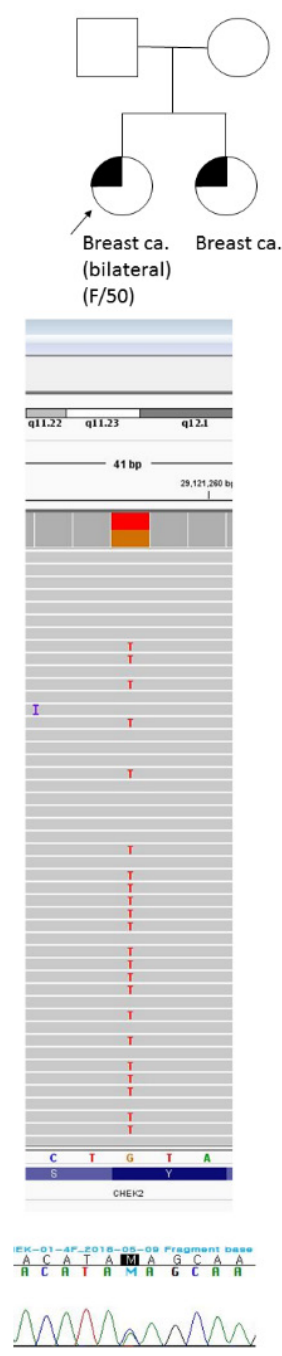

CHEK2 c.546C > A

Pathogenic
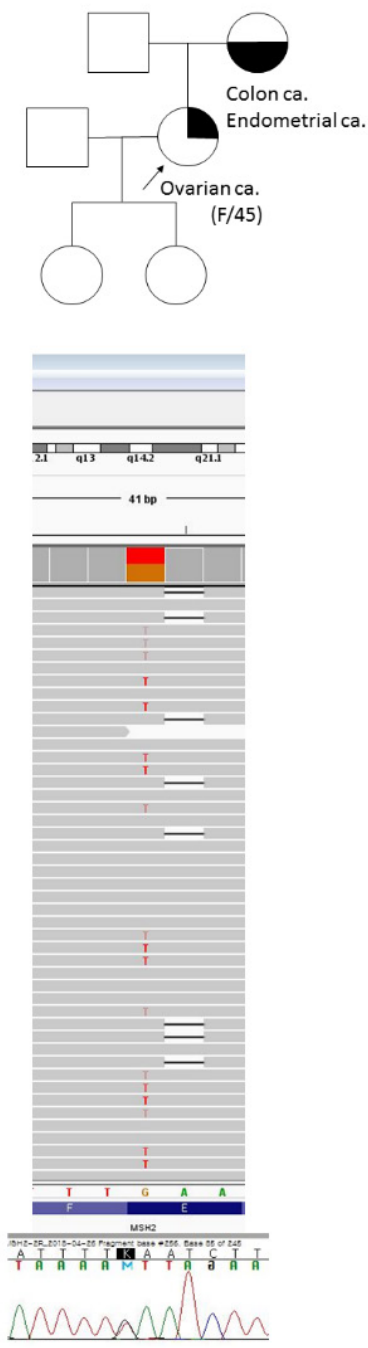

MSH2 c.256G > T
B
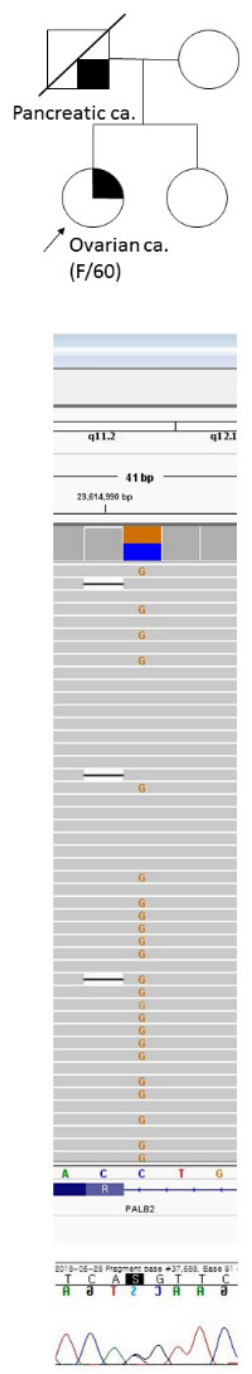

PALB2 c.3351-1G >C Pathogenic
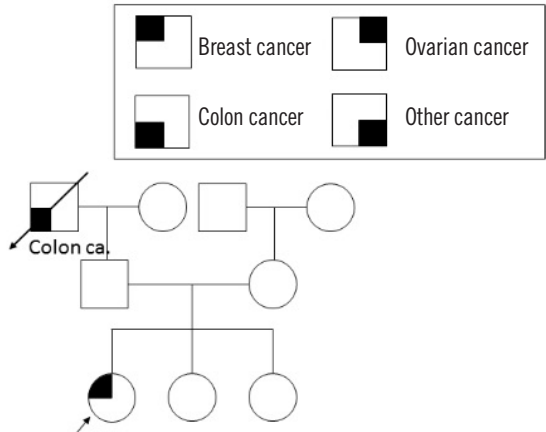

Breast ca.

$(F / 26)$
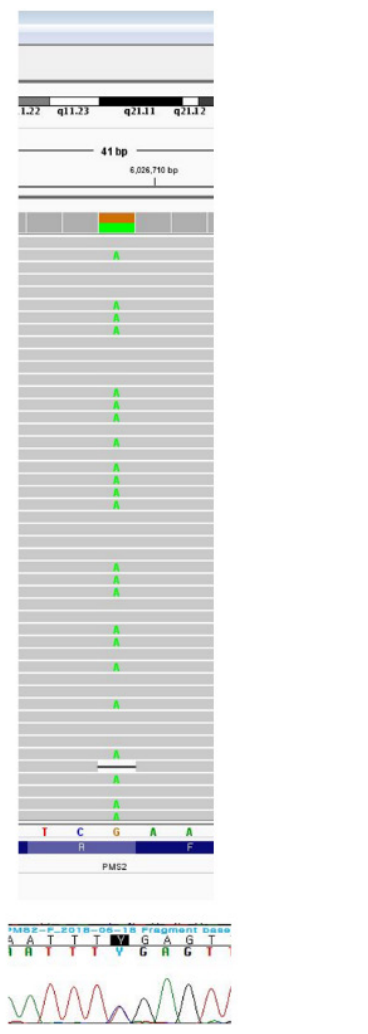

PMS2 c.1687C > T

Pathogenic

Fig. 2. Pedigrees and confirmation in IGV and Sanger sequencing of pathogenic variants in multi-gene panel testing. (A) Pathogenic variant in CHEK2 and MSH2. (B) Pathogenic variants in PALB2 and PMS2.

Abbreviation: IGV, Integrative Genomics Viewer. 


\section{RESULTS}

Pathogenic or likely pathogenic variants in $B R C A 1 / 2$ were detected in 30 patients $(30 / 262,11.5 \%)$. Of the patients included in the multi-gene panel testing group, 3.3\% (4/120) carried a pathogenic variant: two ovarian cancer patients and two breast cancer patients. The median age at diagnosis was 46.5 years (range 26-60 years) (Fig. 2). All four patients had a family history of cancer. The variants identified were MSH2 (c.256G $>$ T, p.Glu86*, pathogenic), PMS2 (c.1687C > T, p.Arg563*, rs587778618, pathogenic), CHEK2 (c.546C >A, p.Tyr182*, rs200917541, pathogenic), and PALB2 (c.3351-1G >C, pathogenic). The variants in MSH2 and PALB2 have not been previously identified. These variants cause premature termination or a splicing error and are classified as pathogenic or likely pathogenic, according to the ACMG criteria (Evidence PVS1, PM1, PM2, PP3-5) [15]. Additionally, a missense variant in the MLH1 gene (c.1937A>G p.Tyr646Cys, rs35045067) of a breast cancer patient was classified as VUS.

Patients with a variant in BRCA1/2 or cancer-related genes ( $N$ =34) had a family history of cancer in a second-degree or closer relative more often than those without a detected variant (40.0\% vs $28.4 \%, P=0.023)$. In one patient with $P M S 2$ variant, the breast cancer recurred one year after surgery and completion of chemotherapy.

\section{DISCUSSION}

We performed a multi-gene panel test using NGS analysis and evaluated its clinical validity in $\mathrm{HBOC}$ patients, focusing on patients negative for pathogenic variants in the BRCA1/2 NGS testing.

$B R C A 1 / 2$ pathogenic variants were detected in $11.5 \%$ of $\mathrm{HBOC}$ patients. This result is lower than that reported by the Korean Hereditary Breast Cancer (KOHBRA) large-cohort study (15.73\%, $378 / 2,403)$ [16]. The difference could be due to the different characteristics of the two studies. The KOHBRA study was a prospective study targeting only breast cancer patients; trained research nurses visited the institution and obtained disease history up to at least three generations back, including third-degree relatives [16]. In contrast, our study targeted breast and ovarian cancer patients and was a retrospective study based on chart review and test results. The advantage of our study is that it reflects real-life clinical situations.

The frequency of pathogenic variants in 25 hereditary cancerrelated genes in our study is in line with previous findings (Table 2) $[10,13$, 17]. All these studies selected $B R C A 1 / 2$-negative breast and/or ovarian cancer patients. Tung, et al. [10] reported that $2.9 \%$ of the 377 patients had pathogenic variants in breast/ ovarian-associated genes but were negative for BRCA1/2 variants. Hirotsu, et al. [13] observed that 1.9\% (3/155) of patients possessed a pathogenic variant in genes that have a central role in DNA repair or the MMR pathway.

The pathogenic variants identified in MSH2, PMS2, and CHEK2 are nonsense variants. MSH2 is an MMR gene, and pathogenic variants in it can lead to Lynch syndrome or hereditary nonpolyposis colorectal cancer syndrome. Nonsense variants, are the most common cause of MMR function deficiency, comprising $82 \%$ of $M S H 2$ pathogenic variants [18]. The patient with $M S H 2$ pathogenic variant had a maternal history of endometrial and colon cancer, suggesting the possibility of developing Lynch syndrome. The c.256G > T (p.Glu86*) variant in MSH2 is novel and was classified as pathogenic according to the ACMG guidelines [15].

The PMS2 gene is also an MMR gene; the PMS2 protein helps correct errors created during DNA duplication. Approximately $2 \%$ of Lynch syndrome families with pathogenic variants have a variant in PMS2 gene [19]. The nonsense variant detected in this study, c.1687C>T (p.Arg563*, rs587778618), is classified as pathogenic in the ClinVar database (http://www.ncbi.nlm.nih. gov/clinvar/) [20-22].

Table 2. Comparison of studies examining breast and/or ovarian cancer patients negative for the $B R C A 1 / 2$ testing

\begin{tabular}{|c|c|c|c|c|}
\hline & This study & Tung, et al. [10] & Hirotsu, et al. [13] & Park, et al. [17] \\
\hline Patients (N) & 120 & 377 & 155 & 120 \\
\hline NGS platform & Ion S5 system (Thermo Fisher) & HiSeq2500 or MiSeq (Illumina) & Ion S5 System (Thermo Fisher) & MiSeq (Illumina) \\
\hline Target genes in panel (N) & 25 & 25 & 25 & 35 \\
\hline Frequency of pathogenic variant (\%) & 3.3 & 4.3 & 1.9 & 7.5 \\
\hline $\begin{array}{l}\text { Genes with identified pathogenic } \\
\text { variant }\end{array}$ & MSH2, PMS2, CHEK2, PALB2 & $\begin{array}{c}\text { CHEK2, ATM, PALB2, BARD1, NBN, } \\
\text { CDH1, MUTYH, APC, CDK2NA }\end{array}$ & ATM, MRE11A, MSH6 & $\begin{array}{l}\text { TP53, PALB2, BARD1, } \\
\text { BRIP1, MRE11A }\end{array}$ \\
\hline Population & Korean & Not disclosed & Japanese & Korean \\
\hline
\end{tabular}

Abbreviation: NGS, next-generation sequencing. 
Checkpoint kinase 2 (CHEK2) is a serine/threonine kinase that is activated on DNA damage and is involved in pathways that activate DNA repair, cell cycle arrest, or apoptosis in response to initial damage [23]. The loss of kinase function has been correlated with different cancer types, mainly breast cancer [23]. The CHEK2 gene is affected by missense or deleterious pathogenic variants. To the best of our knowledge, the CHEK2 c.546C > A variant (p.Tyr182*, rs200917541) is not included in any population databases.

The splicing site variant of the PALB2 gene (c.3351-1G $>C$ ) is classified as pathogenic (PVS1, PM2, and PP3). The Partner and localizer of BRCA2 (PALB2) protein interacts with both BRCA1 and BRCA2, forming a "BRCA complex", in which PALB2 acts as a bridge between BRCA1 and BRCA2. Pathogenic variants in the PALB2 gene are associated with breast, pancreatic, and, possibly, ovarian cancers [24-26].

Additionally, we identified a breast cancer patient with a missense variant in the MLH1 gene (c.1937A > G p.Tyr646Cys, rs35045067). This variant is classified as "uncertain significance" in ClinVar, and is not reported in the Korean reference genome database (KRGDB: http://152.99.75.168/KRGDB/). However, the patient has a significant family history (father: prostate cancer, brother: pancreatic cancer, sister: sarcoma). Thus, further family studies are needed to determine whether this variant is pathogenic or not. The MLH1 gene is another MMR gene implicated in Lynch syndrome. The inclusion of breast cancer within the syndrome remains controversial. Harkness, et al. [27] reported that female $\mathrm{MLH} 1$ carriers appear to be at moderate risk of breast cancer and should be considered for breast screening. Scott, et al. [28] also showed a significant increase in breast cancer cases in an MLH1 pathogenic variant-positive group. Woo, et al. [29] have stated that missense variants in MMR genes should be interpreted with caution and that multiple analyses, including functional analyses, are needed. Thus, further studies, including functional analyses, are needed to refine the clinical significance of this variant and its association with Lynch syndrome.

Our results and previous results demonstrate that there is no prognostic difference between $\mathrm{HBOC}$ patients with and without germline variants of cancer-related genes. Nevertheless, it would be worthwhile to determine the prognostic impact of cancer-related pathogenic variants through a large-cohort study.

Breast or ovarian cancer patients with related pathogenic variants have a family history of not only breast or ovarian cancer but also colon cancer, pancreatic cancer, prostate cancer, and sarcomas. Thus, multi-gene panel testing may be a good screen-

ing tool in such patients with a family history of cancer.

Our study has several limitations. First, family history was not collected sufficiently. To apply NCCN guidelines precisely, information on at least the 2 nd degree family history of patients should be available. Due to the lack of information, some patients might have been misclassified. Second, we did not evaluate all the VUS detected in the multi-gene panel testing. Further evaluation of VUS might affect the prevalence data for the pathogenic variants in $\mathrm{HBOC}$ patients.

In conclusion, the NGS multi-gene panel testing demonstrated significant clinical validity in $\mathrm{HBOC}$ patients as a screening tool, especially for patient with a family history of cancer. The identification of women with pathogenic variants in cancer-related genes may have important implications for family testing.

\section{Author Contributions}

Y.K. and M.K. initiated and designed the study and coordinated the drafting of the manuscript. G.L., J.K., and S.L. performed test and collected data. H.C., J.Y., and E.H. carried out data analysis and wrote the manuscript. All authors read and approved the final manuscript.

\section{Conflicts of Interest}

None declared.

\section{Research Funding}

This study was supported by Research Fund of Seoul St. Mary's Hospital, The Catholic University of Korea.

\section{ORCID}

Jaeeun Yoo Gun Dong Lee Jee Hae Kim Seung Nam Lee Hyojin Chae Eunhee Han Yonggoo Kim Myungshin Kim

https://orcid.org/0000-0001-9917-5393
https://orcid.org/0000-0003-4655-0641
https://orcid.org/0000-0003-2447-9303
https://orcid.org/0000-0002-1862-3666
https://orcid.org/0000-0001-5870-1887
https://orcid.org/0000-0001-6519-1539
https://orcid.org/0000-0003-2808-3795
https://orcid.org/0000-0001-8632-0168

\section{REFERENCES}

1. Smith EC. An overview of hereditary breast and ovarian cancer syndrome. 
J Midwifery Womens Health 2012;57:577-84.

2. Collaborative Group on Hormonal Factors in Breast Cancer. Familial breast cancer: collaborative reanalysis of individual data from 52 epidemiological studies including 58,209 women with breast cancer and 101,986 women without the disease. Lancet 2001;358:1389-99.

3. Crawford B, Adams SB, Sittler T, van den Akker J, Chan S, Leitner O, et al. Multi-gene panel testing for hereditary cancer predisposition in unsolved high-risk breast and ovarian cancer patients. Breast Cancer Res Treat 2017;163:383-90.

4. Couch FJ, Nathanson KL, Offit K. Two decades after BRCA: setting paradigms in personalized cancer care and prevention. Science 2014;343: 1466-70

5. Economopoulou P, Dimitriadis G, Psyrri A. Beyond BRCA: new hereditary breast cancer susceptibility genes. Cancer Treat Rev 2015;41:1-8.

6. Selkirk CG, Vogel KJ, Newlin AC, Weissman SM, Weiss SM, Wang CH, et al. Cancer genetic testing panels for inherited cancer susceptibility: the clinical experience of a large adult genetics practice. Fam Cancer 2014;13:527-36

7. LaDuca H, Stuenkel AJ, Dolinsky JS, Keiles S, Tandy S, Pesaran T, et al. Utilization of multigene panels in hereditary cancer predisposition testing: analysis of more than 2,000 patients. Genet Med 2014;16:830-7.

8. Walsh T, Casadei S, Lee MK, Pennil CC, Nord AS, Thornton AM, et al. Mutations in 12 genes for inherited ovarian, fallopian tube, and peritoneal carcinoma identified by massively parallel sequencing. Proc Natl Acad Sci U S A 2011;108:18032-7.

9. Desmond A, Kurian AW, Gabree M, Mills MA, Anderson MJ, Kobayashi Y, et al. Clinical actionability of multigene panel testing for hereditary breast and ovarian cancer risk assessment. JAMA Oncol 2015;1:943-51.

10. Tung N, Battelli C, Allen B, Kaldate R, Bhatnagar S, Bowles K, et al. Frequency of mutations in individuals with breast cancer referred for $B R C A 1$ and BRCA2 testing using next-generation sequencing with a 25-gene panel. Cancer 2015;121:25-33.

11. Daly MB, Axilbund JE, Buys S, Crawford B, Farrell CD, Friedman S, et al. Genetic/familial high-risk assessment: breast and ovarian. J Natl Compr Canc Netw 2010;8:562-94.

12. Kim DH, Chae H, Jo I, Yoo J, Lee H, Jang W, et al. Identification of large genomic rearrangement of $B R C A 1 / 2$ in high risk patients in Korea. BMC Med Genet 2017;18:38

13. Hirotsu Y, Nakagomi H, Sakamoto I, Amemiya K, Oyama T, Mochizuki H, et al. Multigene panel analysis identified germline mutations of DNA repair genes in breast and ovarian cancer. Mol Genet Genomic Med 2015; 3:459-66.

14. Park J, Jang W, Chae H, Kim Y, Chi HY, Kim M. Comparison of targeted next-generation and sanger sequencing for the BRCA1 and BRCA2 mutation screening. Ann Lab Med 2016;36:197-201.

15. Richards S, Aziz N, Bale S, Bick D, Das S, Gastier-Foster J, et al. Standards and guidelines for the interpretation of sequence variants: a joint consensus recommendation of the American College of Medical Genetics and Genomics and the Association for Molecular Pathology. Genet
Med 2015;17:405-24.

16. Kang E, Seong MW, Park SK, Lee JW, Lee J, Kim LS, et al. The prevalence and spectrum of BRCA1 and BRCA2 mutations in Korean population: recent update of the Korean Hereditary Breast Cancer $(\mathrm{KOH}-$ BRA) study. Breast Cancer Res Treat 2015;151:157-68.

17. Park JS, Lee ST, Nam EJ, Han JW, Lee JY, Kim J, et al. Variants of cancer susceptibility genes in Korean BRCA1/2 mutation-negative patients with high risk for hereditary breast cancer. BMC Cancer 2018;18:83.

18. Peltomaki $\mathrm{P}$ and Vasen H. Mutations associated with HNPCC predisposition -- Update of ICG-HNPCC/INSiGHT mutation database. Dis Markers 2004;20:269-76.

19. Talseth-Palmer BA, McPhillips M, Groombridge C, Spigelman A, Scott RJ. MSH6 and PMS2 mutation positive Australian Lynch syndrome families: novel mutations, cancer risk and age of diagnosis of colorectal cancer. Hered Cancer Clin Pract 2010;8:5.

20. Vasovcak P, Krepelova A, Menigatti M, Puchmajerova A, Skapa P, Augustinakova $A$, et al. Unique mutational profile associated with a loss of TDG expression in the rectal cancer of a patient with a constitutional PMS2 deficiency. DNA Repair (Amst) 2012;11:616-23.

21. Vaughn CP, Robles J, Swensen JJ, Miller CE, Lyon E, Mao R, et al. Clinical analysis of PMS2: mutation detection and avoidance of pseudogenes. Hum Mutat 2010;31:588-93.

22. Goodenberger ML, Thomas BC, Riegert-Johnson D, Boland CR, Plon SE, Clendenning M, et al. PMS2 monoallelic mutation carriers: the known unknown. Genet Med 2016;18:13-9.

23. Apostolou P and Papasotiriou I. Current perspectives on CHEK2 mutations in breast cancer. Breast Cancer (Dove Med Press) 2017;9:331-5.

24. Kluska A, Balabas A, Piatkowska M, Czarny K, Paczkowska K, Nowakowska D, et al. PALB2 mutations in BRCA1/2-mutation negative breast and ovarian cancer patients from Poland. BMC Med Genomics 2017; 10:14.

25. Reid S, Schindler D, Hanenberg H, Barker K, Hanks S, Kalb R, et al. Biallelic mutations in PALB2 cause Fanconi anemia subtype FA-N and predispose to childhood cancer. Nat Genet 2007;39:162-4.

26. Xia B, Sheng Q, Nakanishi K, Ohashi A, Wu J, Christ N, et al. Control of BRCA2 cellular and clinical functions by a nuclear partner, PALB2. Mol Cell 2006;22:719-29.

27. Harkness EF, Barrow E, Newton K, Green K, Clancy T, Lalloo F, et al. Lynch syndrome caused by MLH1 mutations is associated with an increased risk of breast cancer: a cohort study. J Med Genet 2015;52: 553-6.

28. Scott RJ, McPhillips M, Meldrum CJ, Fitzgerald PE, Adams K, Spigelman $\mathrm{AD}$, et al. Hereditary nonpolyposis colorectal cancer in 95 families: differences and similarities between mutation-positive and mutationnegative kindreds. Am J Hum Genet 2001;68:118-27.

29. Woo HI, Woo YM, Kim S, Lee ST, Ki CS, Kim JW. Challenges in assessing pathogenicity based on frequency of variants in mismatch repair genes: an extreme case of a MSH2 variant and a meta-analysis. Gene 2014;546:421-4. 